JOURNAL OF RESEARCH of the National Bureau of Standards-B. Mathematical Sciences

Vol. 79B, Nos. 1 and 2, January-June 1975

\title{
Maximal Network Flows, Matroids and Matchings
}

\author{
Edward Minieka \\ Quantitative Methods Department, University of Illinois, Chicago, Illinois 60680
}

(January 14, 1975)

\begin{abstract}
This paper represents new proofs for some known network flow results. First, maximal network flows are expressed as matroids, and then Gale's characterization of matroids is used to prove the existence of various lexicographic network flows. Second, an independence property of maximum flows is proved and this property is related to an independence property of Brualdi and Scrimger.
\end{abstract}

Key words: Independence properties; matroids; maximal flows; network flows.

\section{Network Flows as Matroids}

This section shows how a network flow may be regarded as a matroid and then shows how Gale's characterization of matroids $[8]^{1}$ can be used to prove the existence of some lexicographic network flows $[7,9]$.

A matroid on a finite set $E$ can be defined [3] as a family $F$ of subsets of set $E$ with the property that

(1) if $A^{\prime} \subseteq A$ and $A \in F$, then $A^{\prime} \in F$, and

(2) for any subset $E^{\prime} \subseteq E$, all maximal members of $F$ that are contained in $E^{\prime}$ have the same cardinality.

This definition is the most appropriate for the purpose of this paper. A survey of other definitions appears in [2].

Let $N=(X, A)$ be a finite, directed network with node set $X$ and arc set $A$. Let $S \subseteq X$ denote the source nodes, and let $T \subseteq X$ denote the sink nodes, where $S \cap T=\phi$. Our interest is in integer valued flows ${ }^{2}$ that are feasible relative to the given integer arc capacities. It is technically convenient to assume that each arc in $A$ has a capacity equal to one and to compensate by assuming that more than one arc may exist between two nodes. (The reader is asked to make the required translations.) Note that with these conventions each flow corresponds to a unique set of arcs (those that carry flow) so that one can speak of maximal flows.

\footnotetext{
AMS(MOS) Subject Classification: 05B35,05C25

' Figures in brackets indicate the literature references at the end of this paper.

2 Once the relationships between networks with integer flows and matroids are understood, the theory can be extended to polymatroids [4] and networks with noninteger capacities and flows. A polymatroid $P$ is a compact nonempty subset of $R_{E}^{+}$(the positive part of real $|E|$ space) such that 
Let $E_{S}$ be the set of all arcs of the form $(x, y), x \in S$, and let $E_{T}$ be the set of all arcs of the form $(x, y), y \in T$. Let $F_{S}$ be the set of all subsets of $E_{S}$ that carry flow in some feasible integer flow in network $N$. Similarly, let $F_{T}$ be the set of all subsets of $E_{T}$ that carry flow in some feasible integer flow in network $N$.

THEOREM 1: The set $\mathrm{F}_{\mathrm{S}}$ is a matroid on set $\mathrm{E}_{\mathrm{S}}$.

PROOF: To see that condition (1) in the definition of a matroid is satisfied, suppose there is a feasible flow in network $N$ that uses to capacity arcs $A \subseteq E_{S}$. Then for any subset $A^{\prime} \subseteq A$, we can construct a feasible flow simply by deleting the units of flow emanating from the arcs in $A-$ $A^{\prime}$. Hence, condition (1) is satisfied.

Let $E_{S}^{\prime}$ be any subset of $E_{S}$. Consider all the maximal members of the set of integer feasibl flows in network $N$ that use only source arcs in set $E_{S}^{\prime}$. Suppose not all these maximal flows have the same value. Then, disregard the arcs in set $E_{S}-E_{S}^{\prime}$, and try to increase the smallest valued maximal flow in the network by using Ford and Fulkerson's flow augmenting algorithm [6], p. 17. It is known that this algorithm must increase the flow on this restricted network (arcs $E_{S}-E_{S}^{\prime}$ are avoided) by an integer amount since the current flow is not maximum on this restricted network. Furthermore, the algorithm does not decrease the flow on any arc adjacent to a source node. Thus all maximal integer flows that use set $E_{S}^{\prime}$ have the same cardinality. Q.E.D.

THEOREM 2: The set $\mathrm{F}_{\mathrm{T}}$ is a matroid on set $\mathrm{E}_{\mathrm{T}}$.

The proof is similar to the proof of Theorem 1 .

Let $F$ be any set of subsets of a totally ordered set $E$. A member $A \in F$ is said to be optimal if for every set $B \in F$, there exists a univalent mapping $f$ from $B$ to $A$ such that $f(b) \geqslant b$ for all $b$ in $B$. Note that set $A$ must be a maximum cardinality member of $F$.

For example, if there is a preference on the order in which the source arcs are to be used, then an optimal flow is a flow that uses the source arcs in the order of greatest preference, i.e., the most preferred source arc if possible, then the second most preferred source arc if possible, etc.

Clearly, not all sets of subsets have an optimal member. For example, a student has a choice between a grade of $A$ in a single course or a grade of $B$ in each of two courses. The set $\{A, B B\}$ possesses no optimal member for the total ordering $A>B$.

THEOREM 3: (Gale) If $\mathrm{F}$ is a matroid, then $\mathrm{F}$ has an optimal member.

The proof of this theorem can be found in [8], p. 178.

THEOREM 4: If the source arcs in a network are ordered, then there exists a maximum flow that uses the source arcs optimally.

The proof is immediate from Theorems 1 and 3. A constructive proof for Theorem 4 that uses only network flow arguments appears in [9].

A dynamic network is a network whose arcs not only have a capacity but also a traverse time, and flow units travel through time in a dynamic network. An earliest departure dynamic flow for $p$ time periods is defined as a flow from the sources to the sinks in a dynamic network in which $V_{i}$ is maximised for each $i=0,1, \ldots, p$, where $V_{i}$ denotes the number of flow units that can leave the sources in the first $i$ time periods. A latest departure dynamic flow for $p$ time periods can be defined as a flow from the sources to the sinks in a dynamic network in which $v_{i}$ is maximised for $i=0,1, \ldots, p$ where $v_{i}$ denotes the number of flow units leaving the sources during the last $i$ time periods, i.e., from time $p-i$ to time $p$. Similarly, an earliest arrival dynamic flow and a latest arrival dynamic flow can be defined. By definition, each of these four types of flows is a maximum flow.

A dynamic network flow for $p$ time periods is equivalent to a flow in a time-expanded, nondynamic replica $N(p)$ of the original dynamic network $N$. In the time expanded network $N(p)$, each node $x$ in network $N$ is represented by $p+1$ nodes $x(0), x(1), \ldots, x(p)$, that represent node $x$ at each time period. The arcs are replicated similarly. See [6], p. 144, for a detailed description of how network $N(p)$ can be constructed from network $N$. Theorem 4 can be applied to network $N(p)$ to show the existence of a maximum flow that uses the source (sink) arcs optimally. 
THEOREM 5: There exists an earliest departure (arrival) dynamic flow in network $\mathrm{N}$ for $\mathrm{p}$ time periods, for $\mathrm{p}=0,1, \ldots$.

PROOF: Form an ordering of all the source (sink) ares in network $N(p)$ as follows: all source (sink) arcs originating from a source $(\operatorname{sink})$ node at time $i$ are in the $i$ th place in the order. By Theorem 4, there exists an optimal, maximal flow on network $N(p)$, and this flow corresponds to an earliest departure (arrival) dynamic flow in the network N. Q.E.D.

A proof for the existence of this flow using network flow techniques first appeared in [7].

THEOREM 6: There exists a latest departure (arrival) dynamic flow in network N.

The proof is similar to the proof for Theorem 5. A proof for this theorem that uses only network flow arguments can be found in [9].

\section{An Independence Property of Network Flows}

This section presents another proof for the independence property of source arcs and sink arcs [9] and shows how this property is related to an independence theorem of Brualdi and Scrimger [1].

THEOREM 7: (Independence of Source Ares and Sink Arcs).

Let $\phi$ and $\theta$ be any two maximal integer flows on a network $\mathrm{N}=(\mathrm{X}, \mathrm{A})$. Let $\mathrm{S}_{\phi} \subseteq \mathrm{E}_{\mathrm{S}}$ and $\mathrm{S}_{\theta}$ $\subseteq \mathrm{E}_{\mathrm{S}}$ denote the source arcs used by flows $\phi$ and $\theta$ respectively. Similarly, let $\mathrm{T}_{\phi} \subseteq \mathrm{E}_{\mathrm{T}}$ and $\mathrm{T}_{\theta} \subseteq$ $\mathrm{E}_{\mathrm{T}}$ denote the sink arcs used by flows $\phi$ and $\theta$ respectively. Then, there exists a flow $\gamma$ such that $\mathrm{S}_{\gamma}=\mathrm{S}_{\phi}$ and $\mathrm{T}_{\gamma}=\mathrm{T}_{\theta}$.

Proof: Let $Z \subseteq A$ denote any minimum cut separating the sources from the sinks in network $N$. We know from the Max Flow-Min Cut Theorem [6], p. 11, that all maximal flows saturate the arcs of cut $Z$. Hence maximal flows $\phi$ and $\theta$ saturate cut $Z$.

Now construct a flow that is identical to flow $\phi$ on all the ares on the "source side" of cut $Z$. This flow, of course, saturates cut $Z$. Then let the flow proceed from cut $Z$ to the sinks along the arcs chosen by flow $\theta$. Clearly, such a construction is possible since cut $Z$ is saturated by flows $\phi$ and $\theta$. The resulting flow satisfies the conditions of the theorem. Q.E.D.

Theorem 7 is called an independence theorem for maximal flows because it demonstrates the independence between the source arcs used by the flow and the sink arcs used by the flow. A constructive proof for Theorem 7 first appeared in [9].

A similar independence theorem appears in [1], p. 253, for matchings. We shall consider only the finite case of this theorem: given two finite sets $S$ and $T$, associate with each $s \in S$ a subset $r(s) \subseteq T$. A matching is defined as any univalent mapping $\phi$ of a subset $S_{\phi}$ of $S$ into $T$ such that if $s \in S_{\phi}$, then $\phi(s) \subseteq r(s)$. A matching $\phi$ is said to be maximal if there is no other matching $\theta$ such that $S_{\phi} \subset S_{\theta}$. Define $T_{\phi}=\phi\left(S_{\phi}\right)$.

THEOREM 8: (Brualdi and Scrimger). Let $\phi$ and $\theta$ be any two maximal matchings between finite sets $\mathrm{S}$ and $\mathrm{T}$. Then there exists a maximal matching $\gamma$ such that $\mathrm{S}_{\gamma}=\mathrm{S}_{\phi}$ and $\mathrm{T}_{\gamma}=\mathrm{T}_{\theta}$.

The proof in [1] uses set theoretic arguments. For the finite case, Theorem 8 is clearly a special case of Theorem 7 for a network $N$ with only source nodes and sink nodes.

Other examples of matching theory results proved by network flow methods can be found in [5].

For dynamic flows, Theorem 7, together with Theorems 5 and 6 , asserts the existence of a maximal dynamic flow that has both a latest departure schedule and an earliest arrival schedule and also the existence of a maximal dynamic flow that has both an earliest departure schedule and a latest arrival schedule. 


\section{References}

[1] Brualdi, R. A. and Scrimger, E. B., Exchange Systems, Matchings and Transversals, J. Combinatorial Theory 5, 244 . 257 (1968).

[2] Bruter, C. P., Vue d'ensemble sur la Theorie des Matroides, Bull. Soc. Math., France, Memoire 17, 48 pages (1969).

[3] Edmonds, J., Minimum Partition of a Matroid into Independent Subsets, J. Res. Nat. Bur. Stand. (U.S.), 69B, 67-72 (1965).

[4] Edmonds, J., Submodular Functions, Matroids, and Certain Polyhedra, Proc. of the Calgary Combinatorial Conference, August 1969, Gordon and Breach, New York, pp. $69-87$ (1970).

[5] Edmonds, J. and Fulkerson, D. R., Transversals and Matroid Partitions, J. Res. Nat. Bur. Stand. (U.S.), 69B, 147153 (1965).

[6] Ford, L. R. and Fulkerson, D. R., Flows in Networks, Princeton University Press, 1962.

[7] Gale, D., Transient Flows in Networks, Michigan Math. J. 6, 59-63 (1959).

[8] Gale, D., Optimal Assignments in an Ordered Set: An Application of Matroid Theory, J. Combinatorial Theory 4. 176-180 (1968).

[9] Minieka, E., Maximal, Lexicographic and Dynamic Network Flows, ORSA, Vol. 20, No. 6, 1162-1170 (1972).

(Paper 79B1\&2-421). 